
\title{
B Reserach S Surare \\ T3 Up-Regulates PDZK1 to Promote the Proliferation of Papillary Thyroid Cancer Cells
}

\author{
Yan Wang \\ Beijing Luhe Hospital \\ Yanan Cheng \\ Beijing Luhe Hospital

\section{Rongxin Sun} \\ Beijing Luhe Hospital \\ Jianan Lang \\ Beijing Luhe Hospital

\section{Longyan Yang} \\ Beijing Luhe Hospital
}

Dong Zhao ( $\nabla$ zhaodong@ccmu.edu.cn )

Beijing Luhe Hospital https://orcid.org/0000-0003-2914-5863

\section{Primary research}

Keywords: papillary thyroid cancer, triiodothyronine, PDZK1, proliferation, invasion

Posted Date: August 3rd, 2021

DOl: https://doi.org/10.21203/rs.3.rs-750790/v1

License: (c) (1) This work is licensed under a Creative Commons Attribution 4.0 International License.

Read Full License 


\section{Abstract}

Aim: Thyroid cancer (TC) is the most common malignant tumor of the endocrine system. Studies have showed that Triiodothyronine (T3) promotes the proliferation of papillary thyroid cancer (PTC) cells, but the specific mechanism remains unclear. Several studies have showed that PDZK1 played important roles in the occurrence and development of cancer. However, the biological function of PDZK1 in PTC remains unclear. Therefore, the aims of this study were to investigate the effect of PDZK1 in PTC and the underlying mechanism.

Materials and Methods: The effect of T3 on proliferation of PTC cell (TPC-1) was analyzed by colony formation assay and real time cell analysis. Immunofluorescence staining aimed to analyze protein expression. RNA-seq was used to analyze the expression of PDZK1. Meanwhile, Western blot was used to verify the protein expression. The effect of PDZK1 on PTC cell proliferation was investigated by Cell Counting Kit-8(CCK8), real time cell analysis, flow cytometry and transwell assay, respectively.

Results: We found that T3 increased the expression of PDZK1 in TPC-1 cells and promote the proliferation of TPC-1 cells which can be weakened after PDZK1 was knocked down. Immunofluorescence staining showed that the expression of PDZK1 was higher in PTC than paracancerous tissues. And the analysis of 6 benign thyroid nodules and 4 thyroid cancer tissues by RNA-Seq showed that the expression of PDZK1 was increased in PTC tissues, the expression of PDZK1 was also increased in PTC cells compared with the normal thyroid epithelium. In addition, PDZK1 promoted TPC-1 cell proliferation was detected by real-time cell analysis (RTCA) and CCK8. Flow cytometry analysis showed that PDZK1 increased cell cycle at S phase and decreased at G1 phase of TPC-1. PDZK1 promoted the invasion of TPC-1 cell was tested by transwell.

Conclusion: These results suggest that T3 can promote the proliferation of PTC cells, which may be mediated by PDZK1. This study illustrates a possible mechanism by which T3 promotes the proliferation of PTC and provides a theoretical basis for the prevention and treatment of PTC.

\section{Introduction}

Thyroid cancer (TC) is the most common malignant tumor of the endocrine system [1]. The number of patients with TC increased at a rate of 3.6\% per year in the United States from 1974 to 2013 [2]. A vast majority of thyroid cancer (approximately $95 \%$ of cases) are derived from thyroid follicular epithelial cells and the remaining $3-5 \%$ of cases originate from parafollicular $\mathrm{C}$ cells [3]. Papillary thyroid cancer (PTC) originated from follicular cells featured by good outcome [4]. However, approximately $30 \%-80 \%$ of PTC patients have central lymph node metastasis [5] and the long-term recurrence rate of PTC is reported to be as high as $30 \%$ [6]. So far, the exact mechanism for this difference is unclear. Therefore, there is an urgent need to better understand the molecular basis of PTC.

Thyroid hormones $(\mathrm{TH})$ are important cellular regulatory factors secreted by thyroid gland, including proliferation, apoptosis, differentiation and metabolism [7]. As the principal TH, Triiodothyronine (T3) 
forms complexes with nuclear TH receptors alpha (TRa) and beta (TRB) [7] and plays an important physiological function [8]. It's reported that T3 played a role in the development of different tumors. Tsui $\mathrm{KH}$ et al. found that $\mathrm{T} 3$ modulated cell proliferation of human prostatic carcinoma cells by determining (3) H-thymidine incorporation [9]. T3 has also been confirmed to regulate actin remodeling and stimulate the migration and invasion of breast cancer cells [10]. T3 induced proliferation in human lung carcinoma cells through estrogen receptor a-dependent and independent pathways [11]. Although there are studies about T3 promoted PTC proliferation $[12,13]$, the exact mechanism is still unclear.

Ferreira $\mathrm{C}$ et al. found that $\mathrm{TH}$ receptors could regulate the expression of PDZ domain-containing 1(PDZK1) [14], PDZK1 (also known as diphor-1, NaPiCap1, CLAMP, CAP70 and NHERF3), as a scaffold protein [15] with four PDZ domains [16], is extensively expressed within organs such as kidney, liver, stomach, skin, et al. [17] PDZK1 could regulate lipid metabolism and tumor progression via interacting with receptor proteins [18]. Increasing evidences have shown that PDZK1 is involved in many tumor types such as renal cancer, gastric cancer and breast cancer. PDZK1 was down-regulated in human clear cell renal cell carcinoma (ccRCC) [18], and inhibited cell proliferation and migration of ccRCC [19]. Our previous study demonstrated that loss of PDZK1 expression contributes to the carcinogenesis and progression of gastric cancer via constitutively activated PTEN-PI3K/AKT signaling [20]. Conversely, PDZK1 possess the function of oncogenicity in breast cancer [21, 22]. These studies indicated that PDZK1 played important roles in regulating the function of tumor cells, and played different or even opposite roles in different tumors. However, the biological function of PDZK1 in PTC remains unclear.

Therefore, this study is aiming to investigate whether T3 influence PTC function by regulating the expression of PDZK1, meanwhile to assess the effect of PDZK1 on PTC proliferation and invasion. This study will great help to better understand the molecular mechanism of PTC and provide a theoretical basis for the prevention and treatment of thyroid cancer.

\section{Materials And Methods}

\subsection{Cell culture and transfection}

Papillary thyroid cancer cell line TPC-1 cell was purchased from the National Infrastructure of Cell Line Resource (Beijing, China). Thyroid epithelium cell line Nthy-ori 3-1 cell was kindly provided by Professor Yang Yan. TPC-1 and Nthy-ori 3 - 1 were grown in Roswell Park Memorial Institute 1640 medium (Gibco) with $10 \%$ fetal bovine serum (FBS, Gibco) and 1\% penicillin/streptomycin (Invitrogen, Carlsbad, CA). Cells were grown in $37^{\circ} \mathrm{C}$ in a humidified atmosphere containing $5 \% \mathrm{CO}_{2}$.

For transfection, cells were plated into six-well plates and cultured in complete RPMI 1640 overnight before transfection. Lipofectamine 2000 (ThermoFisher-Invitrogen, Carlsbad, CA, USA) was used to transfect according to the instructions of manufacturer. Cells were transfected with PDZK1 plasmid to increase the level of PDZK1 and transfected with shPDZK1-1 plasmid or shPDZK1-2 plasmid to decrease the level of PDZK1. Then cells were treated with $10 \% \mathrm{FBS}$ at $37^{\circ} \mathrm{C}$ for indicated times. For stimulation of 
AKT signaling, cells were maintained in FBS-free medium (Life Technologies, Inc., Carlsbad, CA, USA) for 24 hours. The cells were treated with $10 \% \mathrm{FBS}$ at $37^{\circ} \mathrm{C}$ for $15 \mathrm{~min}$. After the medium was removed, cells were harvested in sodium dodecyl sulfate (SDS) sample buffer and analyzed via western blot.

\subsection{Western blot}

Protein expression was detected by immunoblot analysis. Cells were harvested in SDS sample buffer. Samples were separated by $10 \%$ SDS-PAGE and proteins were electro-transferred onto a nitrocellulose membrane using a tank blotting system (Mini Trans-Blot® Cell; Bio-Rad Laboratories AG, Cressier FR, Switzerland).The membrane was blocked in $5 \%$ nonfat dry milk for $1 \mathrm{~h}$ at room temperature and incubated overnight at $4^{\circ} \mathrm{C}$ with the primary antibody: PDZK1 (Abcam) and Myc-Tag (Cell Signaling Technology) at a dilution of 1:1000 in Tris buffered saline supplemented with Tween (TBS-T; 25mM Tris, $140 \mathrm{mM} \mathrm{NaCl}, 2.6 \mathrm{mM} \mathrm{KCl}, 0.4 \%$ Tween 20) with 1\% nonfat dry milk. As loading controls for the cellular fractions the antibodies anti-GAPDH (Santa Cruz Biotechnology) were used at a dilution of 1:3000 in TBS-T with $1 \%$ nonfat dry milk. Horseradish peroxidase-conjugated goat anti-rabbit or goat anti-mouse IgG (1:3000; ORIGENE) was applied as a secondary antibody for $1 \mathrm{~h}$ at room temperature. Membranes were covered with enhanced chemiluminescence solution and exposed to film. Signal intensity was measured using a Bio-Rad XRS chemiluminescence detection system (Bio-Rad, Hercules, CA, USA).

\subsection{Immunofluorescence staining}

Immunofluorescence was performed on paraformaldehyde-fixed paraffin sections. The sections were dewaxed and dehydrated. Rehydration, antigen retrieval in citrate buffer, the sections were blocked for 1 hour using 5\% FBS,then incubated with the primary antibody directed against PDZK1 containing Triton X100 at $4^{\circ} \mathrm{C}$ overnight. After washing 3 times with PBST, sections were incubated with fluorochromeconjugated secondary antibody for 1 hour at room temperature in the dark. After washing 3 times and nuclear staining with DAPI, sections were photographed under a fluorescence microscope (Nanodrop 2000).

\subsection{Cell proliferation assay}

Cells were cultured in 96-well (3000 cells per well) microplates at a density of 2000 cells/well after transfection for 24h. After 0 h, 24 h, 48 h, 72 h and 96h, Cell Counting Kit-8 (CCK-8, Dojindo, Kumamoto, Japan) was added to each well according to the instructions of manufacturer and the cells were cultured for $1 \mathrm{~h}$ at $37^{\circ} \mathrm{C}$. Viable cells were quantified by measuring absorbance at $450 \mathrm{~nm}$ with an EnSpire label microplate reader (PerkinElmer, Waltham, MA). The proliferation assay was repeated three times. Realtime Cell Analysis (RTCA) (ACEA Biosciences, Inc.) was also used to detect cell proliferation assay. 2,000 cells per well were seeded with $100 \mu$ l medium. The effect of PDZK1 on TPC-1 cell proliferation was observed and recorded and the recorded growth curves were shown as cell index SD. Additionally, we tested cell proliferation by colony formation assay. In short, cells were plated (2000 cells/well) in six- well plates. After treatment with T3 for 15 days, colonies were fixed with methanol and stained with $1 \%$ crystal and photographed.

\subsection{Cell cycle assay}


Cells were fixed for overnight in $75 \%$ ethanol at $4^{\circ} \mathrm{C}$ after 24 hours transfection, and then incubated with propidium iodide for $10 \mathrm{~min}$. Flow cytometry (BD Biosciences, San Jose, CA, USA) analysis was used to detect cell cycles. Modfit LT software was used to analyze the data.

\subsection{Invasion assay}

For the invasion assay, $5 \times 10^{4}$ cells were seeded into the upper transwell chambers precoated with Matrigel matrix (BD Biosciences, San Jose, CA) in $100 \mu \mathrm{L}$ RPMI 1640 medium after 24 hours transfection. The lower chamber contained $600 \mu \mathrm{L}$ RPMI 1640 medium with 10\% FBS. After incubation for $24 \mathrm{~h}$, cells that invaded through the chamber membrane were fixed with $4 \%$ formaldehyde solution for $15 \mathrm{~min}$, and were stained with $0.5 \%$ crystal violet solution for $15 \mathrm{~min}$. For each experiment, the numbers of cells in three separate light microscopy fields were counted at 200xmagnifcation. The transwell assay was repeated three times.

\subsection{Statistical analysis}

Data are presented as mean \pm SD. Statistical analysis was performed using SPSS 18.0 (SPSS Inc., Chicago, IL, USA). Two-tailed unpaired Student's t-test was used to determine statistical significance. Statistical significance was accepted for $p<0.05$.

\section{Results}

\subsection{T3 promoted the proliferation and monoclonal formation in PTC cells.}

In order to detect the effect of T3 on the proliferation of PTC cells, we used TPC-1 cells for RTCA assay. TPC-1 cells were stimulated by DMSO $(1 \mathrm{vl} / \mathrm{ml})$ or different concentration of T3 $(0 \mathrm{ng} / \mathrm{ml}, 12.5 \mathrm{ng} / \mathrm{ml}$ or $50 \mathrm{ng} / \mathrm{ml}$ ). And the normalized cell index curves showed that T3 in cell culture medium promoted TPC cell proliferation (Fig. 1A). Next, to verify T3 enhances TPC-1 cells proliferation, colony formation assay was also conducted. In addition, as shown inFigure1B, the clone formation assay further confirmed the longterm proliferative activity of T3 on the proliferation of TPC-1.

\subsection{PDZK1 was required for T3 promoting PTC cell proliferation.}

TR $\beta$, a receptor of T3, up-regulated its downstream molecule PDZK1 [14]. Therefore, we used T3 to stimulate TPC-1 to detect the protein expression of PDZK1. The results showed that T3 promoted the expression of PDZK1 protein in TPC-1 cells (Fig. 2A) and the gray value was shown in Fig. 2B. And then, in order to detect whether T3 can affect cell proliferation by increasing the expression of PDZK1, RTCA and monoclonal formation experiments were carried out after knocking out the PDZK1 of TPC-1 cells. The results showed that after knockout of PDZK1, the promoting effect of T3 on the proliferation and monoclonal formation of TPC-1 cells was weakened (Fig. 2C). 


\subsection{PDZK1 expression was increased in PTC cells and tissues}

In order to detect the expression level of PDZK1 in PTC, we analyzed the levels of PDZK1 in PTC cell line and tissues respectively. First, the protein levels of PDZK1 were detected in human PTC and paracancerous tissues by immunofluorescence staining, and the results showed that the protein level of PDZK1 was higher in PTC than paracancerous tissues (Fig. 4A). Then the protein levels of PDZK1 were analyzed in TPC-1 (PTC cell line) and Nthy-ori $3-1$ (normal follicular cell line)cells, and the results showed that the protein level of PDZK1 was relatively higher in PTC cell as compared with normal thyroid cell (Fig. 4B). Next, to verify whether the levels of PDZK1 were also increased in PTC clinical specimens, we detected the mRNA level of PDZK1 for fine needle aspiration thyroid samples by RNA-seq analysis. Totally 6 cases of benign thyroid node and 4 cases of PTC were collected, and after removing cases with the outlier values of PDZK1 (mean \pm SD), totally 5 cases of benign thyroid node and 4 cases of PTC were included for analysis. It was showed that PDZK1 mRNA was increased in PTC comparing with benign thyroid tissue (Fig. 4C).

\subsection{PDZK1 promoted cell proliferation and cell cycle progression in PTC cells}

Cell proliferation played an important role in PTC development and progression. PDZK1 has been found to regulate proliferation of various cells. To analyze the effects of PDZK1 on PTC cell proliferation, we transfected the myc-PDZK1 plasmid or the corresponding negative control (Vector) into TPC-1 cells (Fig. 4A). And then the transfected TPC-1 cell were plated into 96-well plates and cultured with CCK-8 at 0, $24,48,72$ or 96 hours for 1 hour, separately. As shown in Fig. 4B, the cell proliferation increased in PDZK1 over-expressed group as compared with the control group (Fig. 4B). Cell proliferation ability was also detected by Real-time Cell Analysis, the results showed that PDZK1 high-expressed increased the rate of cell proliferation in TPC-1 cell (Fig. 4C). Next, we transfected two different shPDZK1 plasmids or the corresponding negative control (shRNA) into TPC-1 to decrease the expression of PDZK1 in TPC-1 cell, and the outcome level of PDZK1 was confirmed by western blotting (Fig. 4D). Cell viability was measured, and the PDZK1 low-expressed cells and control cells were cultured in 96-well plates and stained with CCK8 at $72 \mathrm{~h}$, separately. As the result shown, PDZK1 knock-down in TPC-1 cells led to decreased cell viability (Fig. 4E).

To explore the possible mechanism of PDZK1 promoting cell proliferation, we further investigated the effect of PDZK1 on cell cycle progression by using flow cytometry with propidium iodide (PI) staining. Accordingly, after overexpression of PDZK1, the percentage of TPC-1 cells in G0/G1 phase significantly decreased from 49.19-46.15\%, whereas the distribution of cells in S phase increased from 42.8145.85\% (Fig. 5A). By contrast, PDZK1 down-expression could led to the reduction of cells in the $S$ phase and accumulation in G0/G1 phase (Fig. 5B). 


\subsection{PDZK1 increased invasion of TPC-1 cell}

The ability of cell invasion is one important stage in tumor metastasis, therefore we further analyzed the effect of PDZK1 on invasion of TPC-1 by transwell assay. As displayed with staining of cells in matrigelcoated boyden chambers, the TCP-1 cellular invasion was increased after PDZK1 overexpressed (Fig. 6AB). The results indicated that overexpression of PDZK1 increased the ability of the invasion of TPC-1 cell.

\section{Discussion}

This study found that T3 could increase cell proliferation, and the expression of PDZK1 in TPC-1 cells. Increasing evidences have shown that PDZK1 is involved in the biological functions of multiple tumors, such as renal cancer, gastric cancer and breast cancer [19-22]. Therefore we speculated whether T3 exerts its proliferative effect by regulating the expression of PDZK1 in PTC cells. In terms of cytology and histology, we found that PDZK1 was highly expressed in PTC cells and tissues compared with normal cells and paracancerous tissues. Meanwhile overexpression of PDZK1 significantly enhanced the proliferation and invasion in PTC cells. The effect of T3 on PTC proliferation and clone formation was significantly weakened after knockout of PDZK1.These results provide strong evidence for T3 to promote the proliferation and invasion of PTC cells by promoting the expression of PDZK1.

It is reported that T3 plays a different role in different cancers. T3 promoted the proliferation of astrocytoma cells and significantly increased phospho-Akt levels in astrocytoma cells but inhibited the proliferation of glioblastoma cells without ERK or AKT pathway activation[23]. Meng R.et al. found that T3 significantly increased proliferating cell nuclear antigen in non-small cell lung carcinoma and small cell lung cancer and caused phosphorylation of ERa[11]. However, studies have shown T3 can induce colon cancer cells differentiation, accompanied by a decrease in proliferation, which occurred via induction of E-cadherin [24]. In PTC, we found T3 could increase TPC-1 cell proliferation, and Perri A. et al. got the same results that T3 increased thymine incorporation in PTC cell to enhance cell proliferation [13], but the mechanism is not clear.

PDZK1, as a scaffold protein [15], plays dual roles in carcinogenesis depending on the tissue specificity [20-22, 25, 26]. In Gastric Cancer, low levels of PDZK1 led to increase proliferation of gastric cancer cells and poor prognosis of gastric cancer patients with the activation of PI3K/AKT signaling via PTEN phosphorylation [20]. However, it was reported that PDKZ1 stabilized Akt by increasing and interacting with Cdc37 to enhanced cell growth in breast cancer [22]. Hogyoung Kim et al. also found PDZK1 possess the function of oncogenicity in breast cancer requiring insulinlike growth factor 1 receptor expression and function [21]. Similarly, we found that PDZK1 promoted proliferation and invasion in PTC, and after knockout of PDZK1, the growth of PTC was inhibited. The different effects of PDZK1 in tumors may be caused by the heterogeneity.

Although the serine/threonine kinase AKT is a central node in cell signaling downstream of growth factors, cytokines, and other cellular stimuli [27] and active AKT phosphorylates a large and diverse array 
of down-stream substrates [28] promoting cell survival and growth in cellular metabolic pathways [29], the biological role of PDZK1 on PTC is not mediated by PI3K/AKT signaling pathway (Supplement Fig. 1). PDZK1 may regulate the biological function of PTC through other signaling pathways of MAPK, which we will continue to explore.

\section{Conclusions}

In conclusion, our study found that T3 could promote the proliferation of PTC, which may be mediated by PDZK1. This study elucidated a possible mechanism of T3 promoting the proliferation of thyroid cancer and provided a theoretical basis for the prevention and treatment of thyroid cancer.

\section{Abbreviations}

TC: Thyroid cancer; T3: Triiodothyronine; PTC: papillary thyroid cancer; CCK8: Cell Counting Kit-8; RTCA: real-time cell analysis; TH: Thyroid hormone; TRa: thyroid hormone receptors alpha; TRß: thyroid hormone receptors beta; PDZK1: PDZ domain-containing 1; ccRCC: clear cell renal cell carcinoma; FBS: fetal bovine serum; SDS: sodium dodecyl sulfate; DAPI: 4',6-diamidino-2-phenylindole; SD: Standard deviation; OD: optical density.

\section{Declarations}

\section{Author contributions}

These authors were involved with this manuscript: Dong Zhao, Longyan Yang(study concept and design, drafting of the manuscript); Yan Wang, Yanan Cheng,(acquisition of data; analysis and interpretation of data; statistical analysis); All authors contributed to the reviewing of the manuscript, and approved the final manuscript for submission.

\section{Acknowledgements}

Sincere thanks for all supports.

\section{Conflict of interest}

The authors declare that they have no conflict of interest.

\section{Availability of data and materials}

Relevant data and materials during this study are included in this published article and its supplementary information files. 
This research did not receive any specific grant from funding agencies in the public,commercial, or notfor-profit sectors.

\section{Consent for publication}

All listed authors have actively participated in the study and have read and approved the submitted manuscript.

\section{Ethics approval and consent to participate}

The study complied with the Helsinki Declaration for investigation of human subjects. Ethical approval was obtained from the competent Institutional Review Boards of the Lu He hospital and Capital Medical University. All patients provided written informed consent.

\section{References}

1. Kilfoy B, et al., Gender is an age-specific effect modifier for papillary cancers of the thyroid gland. 2009. 18(4): p. 1092-100.http://doi.org/10.1158/1055-9965.Epi-08-0976.

2. Lim H, et al., Trends in Thyroid Cancer Incidence and Mortality in the United States, 1974-2013. 2017. 317(13): p. 1338-1348.http://doi.org/10.1001/jama.2017.2719.

3. Mariniello R, et al., The TUSC2 Tumour Suppressor Inhibits the Malignant Phenotype of Human Thyroid Cancer Cells via SMAC/DIABLO Protein. 2020. 21(3).http://doi.org/10.3390/ijms21030702.

4. Wang T, Sosa JJNrE. Thyroid surgery for differentiated thyroid cancer - recent advances and future directions. 2018. 14(11): p. 670-683.http://doi.org/10.1038/s41574-018-0080-7.

5. Lee Y, et al., Risk factors for recurrence in patients with papillary thyroid carcinoma undergoing modified radical neck dissection. 2016. 103(8): p. 1020-5.http://doi.org/10.1002/bjs.10144.

6. Pastorčić Grgić M, et al., Total Thyroidectomy with Central Node Dissection is a Valuable Option in Papillary Thyroid Cancer Treatment. 2020. 59: p. 102107.http://doi.org/10.20471/acc.2020.59.s1.13.

7. Liu F, et al., Effects of gold nanorods modified with antiepidermal growth factor receptor monoclonal antibody on laryngeal cancer cells. 2018. 42(2): p. 144-151.http://doi.org/10.3906/biy-1708-101.

8. Foster J, Tinwell H. and S.J.A.o.t. Melching-Kollmuss, A review of species differences in the control of, and response to, chemical-induced thyroid hormone perturbations leading to thyroid cancer. 2021. 95(3): p. 807-836.http://doi.org/10.1007/s00204-020-02961-6.

9. Tsui $\mathrm{K}$, et al., Triiodothyronine modulates cell proliferation of human prostatic carcinoma cells by downregulation of the B-cell translocation gene 2. 2008. 68(6): p. 6109.http://doi.org/10.1002/pros.20725.

10. Flamini $\mathrm{M}$, et al., Thyroid Hormone Controls Breast Cancer Cell Movement via Integrin aV/ B3/SRC/FAK/PI3-Kinases. 2017. 8(1): p. 16-27.http://doi.org/10.1007/s12672-016-0280-3. 
11. Meng R, et al., Crosstalk between integrin av $\beta 3$ and estrogen receptor-a is involved in thyroid hormone-induced proliferation in human lung carcinoma cells. 2011. 6(11):

p. e27547.http://doi.org/10.1371/journal.pone.0027547.

12. Lin $\mathrm{H}$, et al., Thyroid hormone is a MAPK-dependent growth factor for thyroid cancer cells and is antiapoptotic. 2007. 72(2): p. 180-7.http://doi.org/10.1016/j.steroids.2006.11.014.

13. Perri A, et al., T3 enhances thyroid cancer cell proliferation through TRB1/Oct-1-mediated cyclin D1 activation. 2014. 382(1): p. 205-217.http://doi.org/10.1016/j.mce.2013.10.001.

14. Ferreira $\mathrm{C}$, et al., $P D Z$ domain containing protein 1 (PDZK1), a modulator of membrane proteins, is regulated by the nuclear receptor THRß. 2018. 461: p. 215225.http://doi.org/10.1016/j.mce.2017.09.017.

15. Ferreira $\mathrm{C}$, et al., The scaffold protein PDZK1 modulates expression and function of the organic anion transporting polypeptide 2B1. 2018. 120: p. 181-190.http://doi.org/10.1016/j.ejps.2018.05.006.

16. Shimizu T, et al., PDZK1 regulates breast cancer resistance protein in small intestine. 2011. 39(11): p. 2148-54.http://doi.org/10.1124/dmd.111.040295.

17. Kocher $\mathrm{O}$, et al., Identification and partial characterization of PDZK1: a novel protein containing PDZ interaction domains. 1998. 78(1): p. 117-25.

18. Tao T, et al., PDZK1 inhibits the development and progression of renal cell carcinoma by suppression of SHP-1 phosphorylation. 2017. 36(44): p. 6119-6131.http://doi.org/10.1038/onc.2017.199.

19. Qi Y, et al., Long noncoding RNA PENG upregulates PDZK1 expression by sponging miR-15b to suppress clear cell renal cell carcinoma cell proliferation. 2020. 39(22): p. 44044420.http://doi.org/10.1038/s41388-020-1297-1.

20. Zhao $\mathrm{C}$, et al., Loss of PDZK1 expression activates PI3K/AKT signaling via PTEN phosphorylation in gastric cancer. 2019. 453: p. 107-121.http://doi.org/10.1016/j.canlet.2019.03.043.

21. $\mathrm{Kim} \mathrm{H}$, et al., PDZK1 is a novel factor in breast cancer that is indirectly regulated by estrogen through IGF-1R and promotes estrogen-mediated growth. 2013. 19: p. 253 62.http://doi.org/10.2119/molmed.2011.00001.

22. Kim H, et al., Correlation between PDZK1, Cdc37, Akt and breast cancer malignancy: the role of PDZK1 in cell growth through Akt stabilization by increasing and interacting with Cdc37. 2014. 20: p. 270-9.http://doi.org/10.2119/molmed.2013.00166.

23. Liappas A, et al., Cell-type-dependent thyroid hormone effects on glioma tumor cell lines. 2011. 2011: p. 856050.http://doi.org/10.4061/2011/856050.

24. Dentice $\mathrm{M}$, et al., $\beta$-Catenin regulates deiodinase levels and thyroid hormone signaling in colon cancer cells. 2012. 143(4): p. 1037-47.http://doi.org/10.1053/j.gastro.2012.06.042.

25. Zheng J, et al., Low level of PDZ domain containing 1 (PDZK1) predicts poor clinical outcome in patients with clear cell renal cell carcinoma. 2017. 15: p. 6272.http://doi.org/10.1016/j.ebiom.2016.12.003. 
26. Inoue J, et al., Overexpression of PDZK1 within the 1q12-q22 amplicon is likely to be associated with drug-resistance phenotype in multiple myeloma. 2004. 165(1): p. 7181.http://doi.org/10.1016/s0002-9440(10)63276-2.

27. Manning B, Cantley LJC, AKT/PKB signaling: navigating downstream. 2007. 129(7): p. 126174.http://doi.org/10.1016/j.cell.2007.06.009.

28. Hoxhaj G, Manning BJNrC, The PI3K-AKT network at the interface of oncogenic signalling and cancer metabolism. 2020. 20(2): p. 74-88.http://doi.org/10.1038/s41568-019-0216-7.

29. Manning B, Toker AJC. AKT/PKB Signaling: Navigating the Network. 2017;169(3):381-405. http://doi.org/10.1016/j.cell.2017.04.001.

\section{Figures}

\section{Fig.1}

A

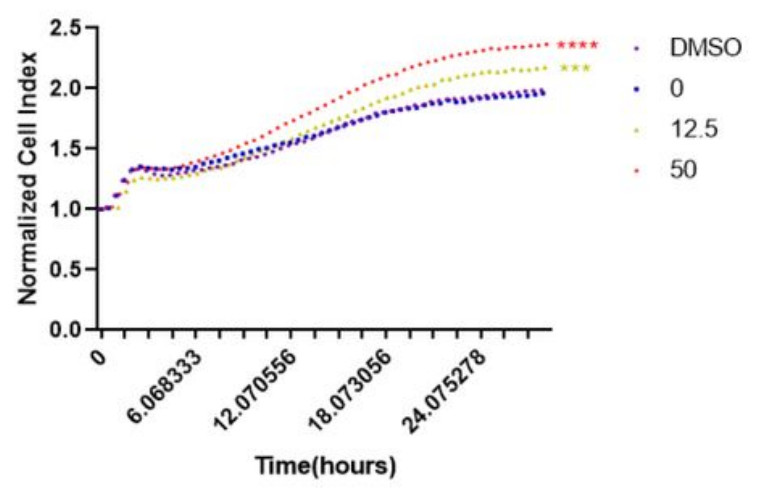

B

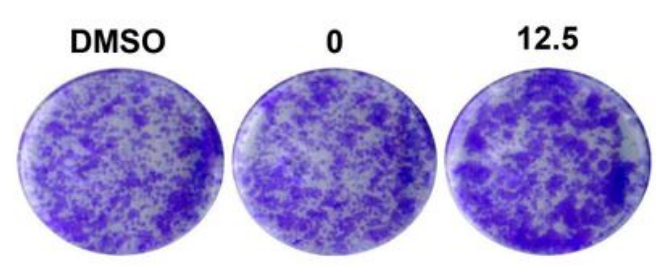

\section{Figure 1}

T3 promotes the proliferation of TPC-1 cells. A. B. Colony formation assay and Real-time Cell Analysis were used to investigate the effect of T3 on the proliferation of TPC-1 cells $₫ P<0.05 \rrbracket$. 
Fig.2

A

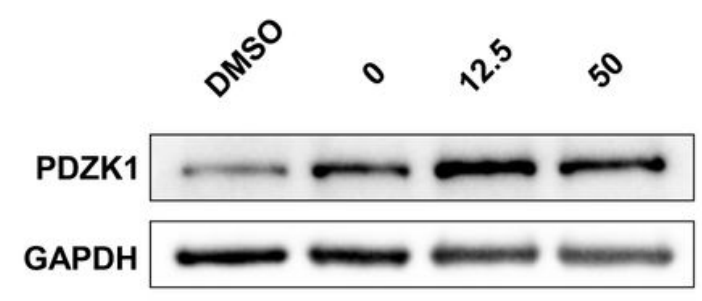

C

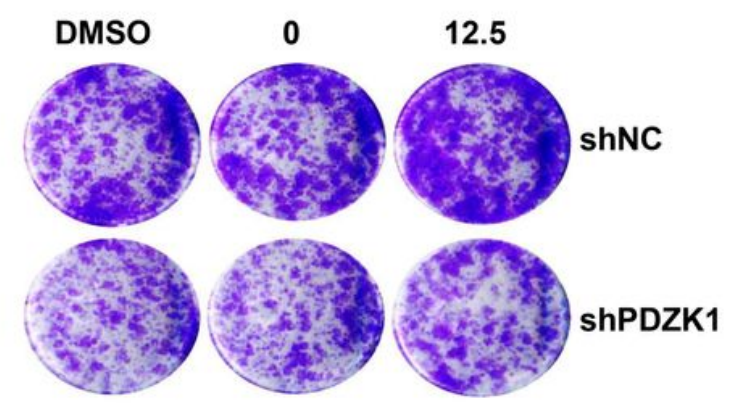

B

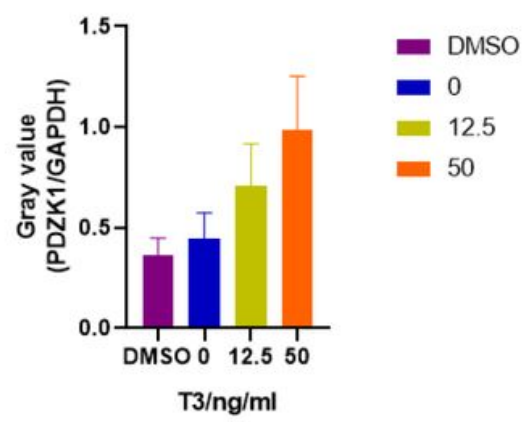

\section{Figure 2}

PDZK1 is required for T3 promoting PTC cell proliferation. A.B. PDZK1 protein levels in TPC-1 cells after stimulated by T3 or DMSO were analyzed by immunohistochemistry and the gray value of PDZK1/GAPDH was analyzed. C. Colony formation assay was used to investigate the effect of T3 on the proliferation of TPC-1 cells after PDZK1 knockdown. 
Fig.3

A
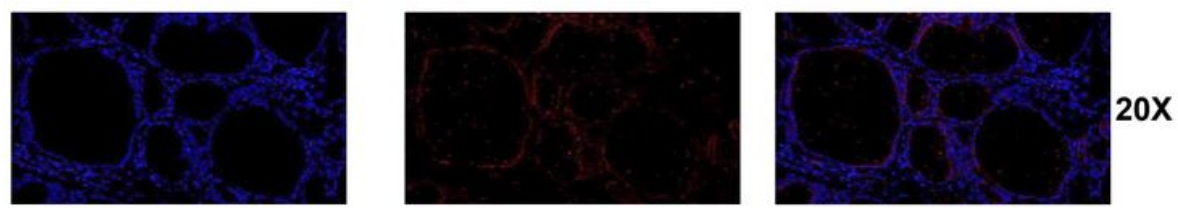

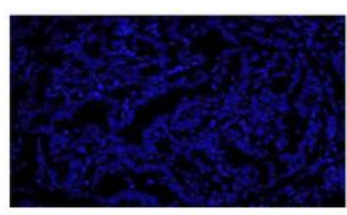

DAPI

B

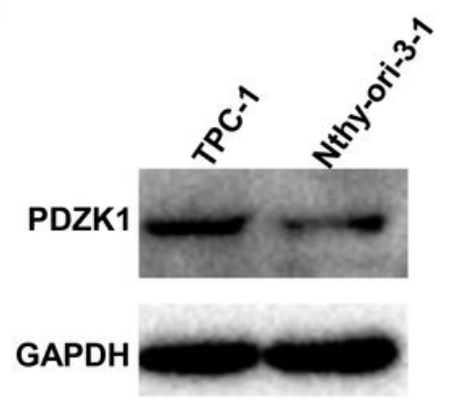

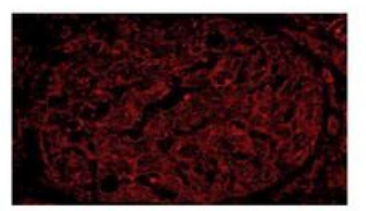

PDZK1

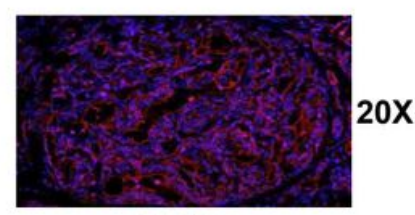

Merge

C

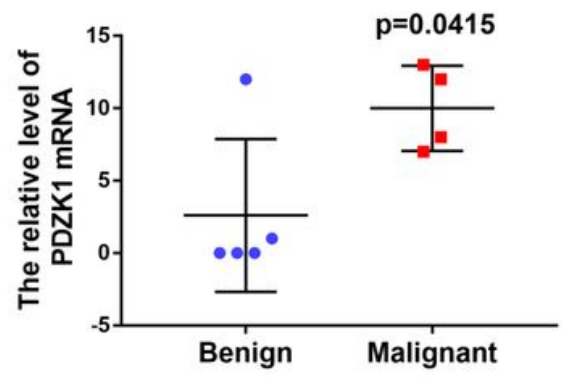

Figure 3

PDZK1 expression was increased in PTC cells and tissues. A. PDZK1 protein levels in thyroid carcinoma and paracancerous tissues were detected by immunofluorescence staining. B. PDZK1 protein levels in TPC-1 cells and Nthy-ori-3-1 cells were analyzed by western blot. C.PDZK1 mRNA expression level was higher in thyroid cancer than benign thyroid tissue $(\mathrm{p}<0.05)$. 
Fig.4

A

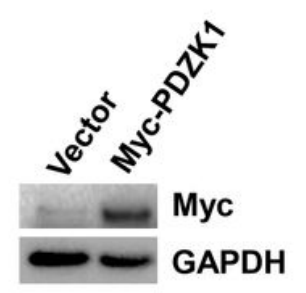

D

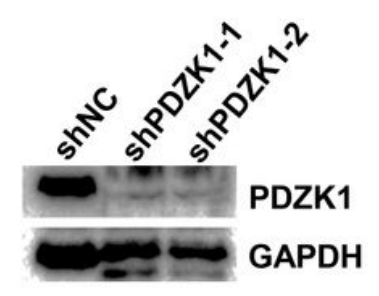

B

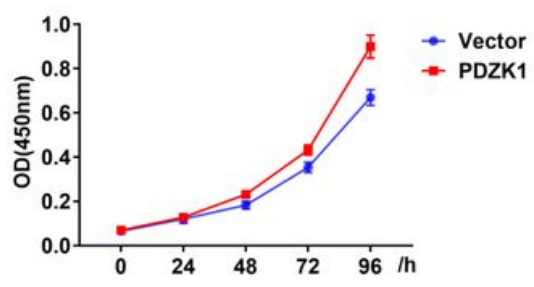

E

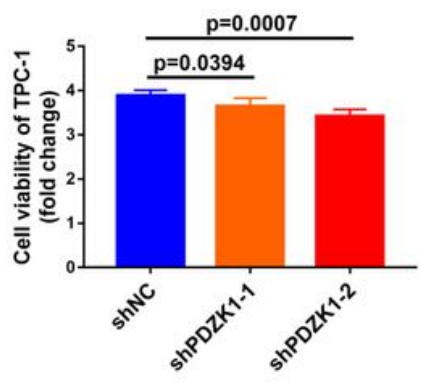

C

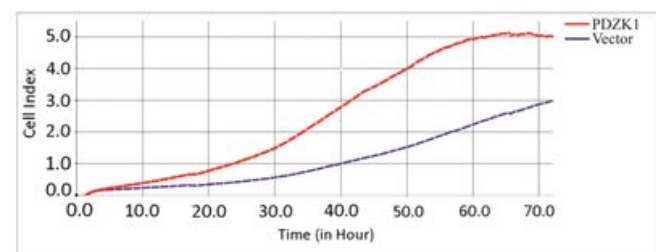

\section{Figure 4}

PDZK1 increases cell proliferation on papillary thyroid cancer. A. Overexpression of PDZK1 in TPC-1 cells verified by western blot analysis; GAPDH was used as a loading control. B. CCK8 assay was performed to assess the proliferation rate of TPC-1 after Myc-PDZK1 or Vector transfection. Absorbance at O.D. 450 $\mathrm{nm}$ was measured every $24 \mathrm{~h}$ for four days. C. Real-time Cell Analysis was performed to assess the proliferation rate of TPC-1 after Myc-PDZK1 or Vector transfection. D. PDZK1 knockdown by shPDZK1 in TPC-1 cells was confirmed by western blot analysis. E. Knockdown of PDZK1 reduced the cell viability in TPC-1 cells $(p<0.05)$. 
Fig.5

A

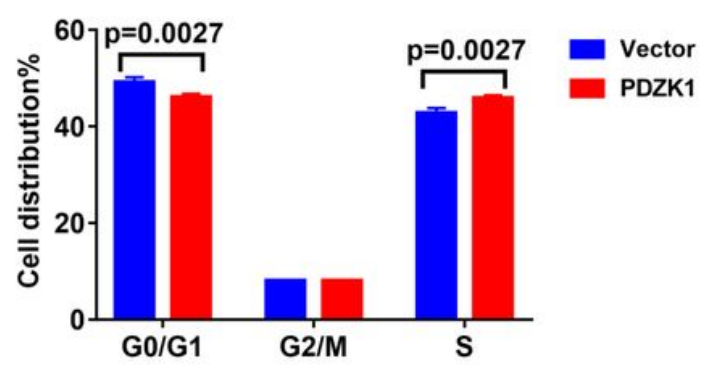

B

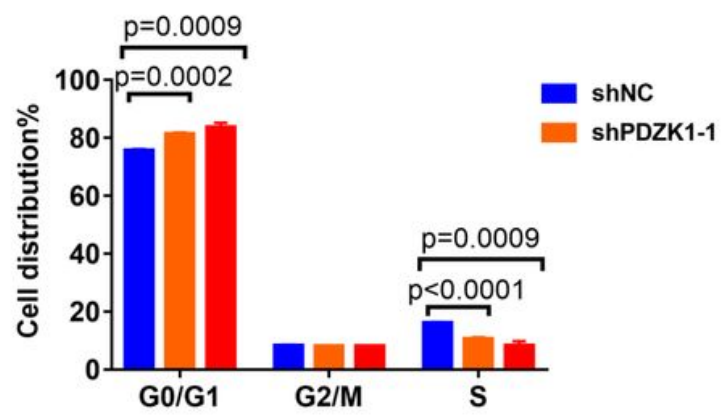

Figure 5

PDZK1 expression influenced the cell cycle of thyroid cancer cell. A. Cell cycle progression was analyzed by flow cytometry in PDZK1 overexpressed TPC-1. B. Cell cycle progression was analyzed by flow cytometry in PDZK1 down-expressed TPC-1. 
Fig.6

A

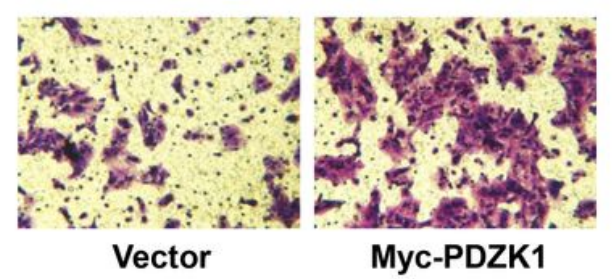

B

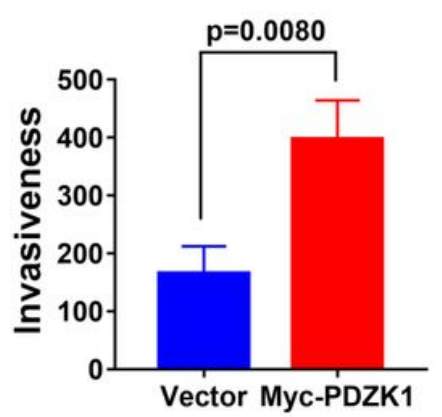

\section{Figure 6}

PDZK1 increased cell invasion on thyroid cancer cell. A. Overexpression of PDZK1 enhanced cell invasion. The Boyden chambers invasion assay was used. B. The statistic result of cell number per well after washing out the crystal violet.

\section{Supplementary Files}

This is a list of supplementary files associated with this preprint. Click to download.

- Additionalfile1.jpg 